\title{
MIXED NON-ALBICANS CANDIDA FUNGEMIA: AN UNCOMMON BUT EASILY OVERLOOKED CLINICAL ENTITY
}

\author{
CHUAN HUN DING*, ZAILI ZAKI
}

Department of Medical Microbiology and Immunology, Faculty of Medicine, Universiti Kebangsaan Malaysia, Kuala Lumpur, Malaysia. Email: dingch@ppukm.ukm.edu.my

Received: 20 July 2018, Revised and Accepted: 20 August 2018

\begin{abstract}
The increasing use of invasive medical devices and broad-spectrum antimicrobial agents has resulted in rising candidemia rates throughout the world. A 70-year-old diabetic and schizophrenic woman was admitted initially for staphylococcal sepsis secondary to an infected sacral sore but developed a catheter-related bloodstream infection caused by extended-spectrum $\beta$-lactamase-producing Klebsiella sp. which necessitated the administration of meropenem. Unfortunately, after a week on the carbapenem, the bacterial sepsis was followed by candidemia. Parenteral fluconazole therapy was started pending identification of the yeast(s). Two distinct Candida species were isolated from her blood which were identified biochemically using ID $32 \mathrm{C}$ as Candida tropicalis and Candida lipolytica. Both yeasts possessed elevated minimal inhibitory concentrations toward fluconazole and although amphotericin B was eventually administered, the patient succumbed to her illness.
\end{abstract}

Keywords: Candida lipolytica, Candida tropicalis, Fluconazole, Non-albicans Candida.

(C) 2018 The Authors. Published by Innovare Academic Sciences Pvt Ltd. This is an open access article under the CC BY license (http://creativecommons. org/licenses/by/4. 0/) DOI: http://dx.doi.org/10.22159/ajpcr.2018.v11i11.28612

\section{INTRODUCTION}

Among the fungi of medical importance, Candida spp. have been implicated in up to $90 \%$ of invasive fungal infections [1]. Knowing the precise species of Candida is not merely of academic interest because the mortality rate associated with candidemia is also higher when caused by non-albicans Candida (NAC) [2]. This observation is attributable to the fact that NAC are notoriously less susceptible to fluconazole than C. albicans [3]. Fluconazole has been favored over antifungals from other classes due to its relatively low-cost, good tolerability, and favorable side effect profile. Thus, the usage of antifungal agents from other classes (e.g. polyenes) could potentially expose patients to more adverse drug effects. To compound matters, the proportion of NAC species causing candidemia has been rising steadily in recent years and has in fact overtaken $C$. albicans as the most common cause of candidemia in some centers [4]. This case report discusses the risk factors for NAC fungemia as well as its management.

\section{CASE REPORT}

A 70-year-old woman with schizophrenia and long-standing diabetes mellitus was brought to Universiti Kebangsaan Malaysia Medical Centre for high-grade fever for 2 days. For the past 1 year, she has been suffering from antipsychotic-induced parkinsonism and has been bedbound and dependent on family members for activities of daily living since then. On examination, her Glasgow Coma Scale score was 10/15 and she appeared dehydrated. Her blood pressure was 106/46 $\mathrm{mmHg}$, pulse rate 97 beats per minute, and body temperature $38.2^{\circ} \mathrm{C}$ and her oxygen saturation on room air was $97 \%$. There were fixed flexion deformities on both upper limbs and a Grade 3 ulcer was seen over the sacral region. The sacral sore contained necrotic tissue and exuded a foul-smelling yellowish discharge. Lung, abdominal, and cardiovascular examinations were unremarkable.

Preliminary laboratory investigations revealed a high random blood glucose level (28 mmol/L), mild anemia (hemoglobin level of $10.7 \mathrm{~g} / \mathrm{dL}$ ), mild leukocytosis (total white cell count of $14 \times 10^{9} / \mathrm{L}$ ), as well as raised serum urea and creatinine levels $(31 \mathrm{mmol} / \mathrm{L}$ and $163 \mu \mathrm{mol} / \mathrm{L}$, respectively). She had metabolic acidosis with a blood $\mathrm{pH}$ of 7.36, $\mathrm{PCO}_{2}$ of $31.3 \mathrm{mmHg}$, and a bicarbonate level of $18.5 \mathrm{mmol} / \mathrm{L}$. Her urine was negative for ketones. Her initial blood culture grew methicillin-susceptible Staphylococcus aureus.

She was initially diagnosed with bacterial sepsis secondary to an infected sacral sore. Intravenous (IV) cloxacillin $1 \mathrm{~g}$ q8h was started along with insulin therapy. A right triple lumen femoral catheter was inserted due to difficult peripheral venous access for administration of antibiotics. However, while being treated for staphylococcal bacteremia, the patient developed another spike of temperature. New blood culture specimens were taken from both the femoral catheter and a peripheral vein. Both central and peripheral blood cultures yielded extendedspectrum $\beta$-lactamase-producing Klebsiella sp., with a differential time to positivity of at least 2 hours. The femoral catheter was subsequently removed and IV meropenem $1 \mathrm{~g}$ q8h was commenced.

After a week on meropenem, the patient's clinical condition did not appear to have improved and a third blood culture was taken. This time, budding yeast cells were seen on the Gram stain of the positive blood culture. A statim dose of IV fluconazole $600 \mathrm{mg}$ followed by daily doses of $300 \mathrm{mg}$ were administered as empirical therapy while awaiting formal identification of the yeast. In the mycology laboratory, the yeast was subcultured onto CHROMagar Candida plates, and colonies of two distinct colors were formed (i.e., blue and grayish-white) as shown in Fig. 1.

The two yeasts were identified using a commercial biochemical identification kit, ID 32 C (bioMérieux, France). The yeast which formed blue colonies was identified as Candida tropicalis (numerical profile 5167340115; 95.4\% identification) while the yeast which grew as whitish colonies was identified as Candida lipolytica (numerical profile 2300511211; 99.9\% identification). Antifungal susceptibility testing was performed on both Candida species with the broth microdilution method (Sensititre ${ }^{\circledR}$ YeastOne $₫$ Y010) (TREK Diagnostic Systems, USA). The minimal inhibitory concentration (MIC) values were read at $24 \mathrm{~h}$ and are shown in Table 1. Following the release of the laboratory report, fluconazole therapy was discontinued immediately and IV amphotericin B at a dose of $0.5 \mathrm{mg} / \mathrm{kg} /$ day was started. Unfortunately, the patient succumbed to her illness. 


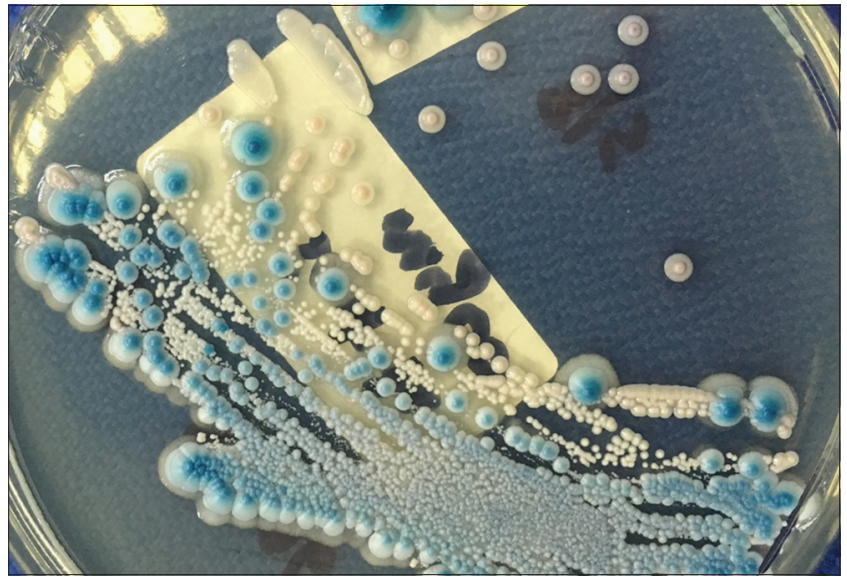

Fig. 1: Distinct bluish and grayish-white yeast colonies on a CHROMagar Candida plate

Table 1: MIC values for C. tropicalis and C. lipolytica

\begin{tabular}{lll}
\hline $\begin{array}{l}\text { Antifungal } \\
\text { agent }\end{array}$ & $\begin{array}{l}\text { C. tropicalis } \text { MIC } \\
(\boldsymbol{\mu g} / \mathbf{m L})\end{array}$ & $\begin{array}{l}\text { C. lipolytica MIC } \\
(\boldsymbol{\mu g} / \mathbf{m L})\end{array}$ \\
\hline Anidulafungin & 0.12 & 0.25 \\
Micafungin & 0.03 & 0.5 \\
Caspofungin & 0.03 & 0.25 \\
Flucytosine & 0.06 & 0.5 \\
Posaconazole & 1 & 2 \\
Voriconazole & 8 & 1 \\
Itraconazole & 1 & 1 \\
Fluconazole & 128 & 64 \\
Amphotericin B & 1 & 0.5 \\
\hline
\end{tabular}

MIC: Minimal inhibitory concentration, C. tropicalis: Candida tropicalis, C. lipolytica: Candida lipolytica

\section{DISCUSSION}

In general, among the NAC species, $C$. tropicalis is one of the three most frequently isolated species in candidemia, with the other two being C. parapsilosis and C. glabrata [4]. In India, C. tropicalis has the distinction of being the most common infecting species [5]. On the other hand, C. lipolytica is a relatively obscure NAC species considered to be of low virulence, but it is emerging as an important cause of nosocomial infections [6]. Both NAC species isolated from our patient were fluconazole resistant, in keeping with the belief that NAC are generally less susceptible to fluconazole. It is interesting to note that two different NAC species were isolated from the same specimen. This finding could have been missed if we had not been utilizing chromogenic agars (CHROMagar Candida) for routine yeast subcultures in our laboratory. CHROMagar Candida has two main benefits over the standard Sabouraud dextrose agars in being able to detect mixed cultures and provide presumptive identification for $C$. albicans. Colonies which are not green on CHROMagar Candida can be presumptively considered to be NAC and subjected to further identification [7].

Like bacteremia, fungemia has also been traditionally regarded as a monomicrobial disease, and the isolation of more than one fungal species from a blood specimen is an uncommon occurrence [8]. Published figures on mixed fungemia vary slightly from region to region, with Spanish investigators reporting an incidence of $2.8 \%$, Canadian investigators $4 \%$, and the United States investigators 5.2\% [8-10]. However, in all three studies, C. albicans was isolated in more than half of the mixed fungemia cases. Thus, the incidence of true mixed NAC fungemia is expected to be a lot lower. Since it has already been established that invasive monomicrobial NAC infections carry a higher mortality than that caused by $C$. albicans alone, it is worthy to know if morbidity/mortality rates for mixed candidemia (in which at least one of the organisms is a NAC) would also be amplified. Surprisingly, it has been reported that mixed candidemia is not associated with a more severe outcome compared to monomicrobial candidemia [8]. However, whether this finding can be applied to patients with mixed $C$. tropicalis and C. lipolytica fungemia remains to be ascertained because at the time of writing, there are no data in the published literature on this specific species combination.

Although there are several risk factors known to be associated with candidemia, it is worthwhile to explore if any of these risk factors, particularly predispose patients to NAC fungemia. Considering that NAC are often less susceptible to fluconazole, it is logical to regard prior fluconazole usage as the main risk factor although this was clearly not the case with our patient who was on a broad-spectrum antibiotic (i.e., meropenem) rather than an antifungal before the candidemia. Broad-spectrum antibiotic usage is a non-specific risk factor for candidemia, including that caused by C. albicans [9]. On the contrary, specific risk factors for NAC fungemia are neutropenia, acute leukemia, and other hematological malignancies, prior surgery, catheter insertion, and renal failure [4]. Our patient had an indwelling femoral catheter and renal impairment (as evidenced by her high serum urea and creatinine levels) before the onset of the NAC fungemia. However, due to the paucity of clinical data in the medical literature, it remains to be corroborated if the insertion of a vascular catheter in a patient with underlying renal impairment will predispose him/her to mixed C. tropicalis and C. lipolytica fungemia.

Once a diagnosis of NAC fungemia is established, the next course of action is prompt and optimal treatment. The two key approaches recommended by the Infectious Diseases Society of America in their 2016 guideline on the management of candidiasis are administering an antifungal agent and removing the central venous catheter as early as possible. An echinocandin is recommended as the initial therapy, with fluconazole and amphotericin B being acceptable alternatives [11]. However, the guideline further states that if fluconazole is to be administered, the infecting Candida species should be one which is unlikely to be fluconazole resistant. Thus, the role of in vitro antifungal susceptibility testing is crucial, particularly so when faced with NAC species with unpredictable fluconazole susceptibility, as documented here. Perhaps, new sources or classes of antifungal agents need to be sought and evaluated for human use as well $[12,13]$. With regard to catheter removal, its role in the management of candidemia is especially imperative if it is the source of infection. In fact, for some patients with $C$. lipolytica fungemia, catheter removal alone (i.e., without the administration of any antifungal agent) has been sufficient in clearing the fungemia [6].

\section{CONCLUSION}

Both clinicians and laboratorians need to be aware of mixed NAC fungemia, particularly in patients who have indwelling central venous catheters and other risk factors for candidemia such as renal impairment and broad-spectrum antibiotic usage. Clinical mycology laboratories are advised to routinely use chromogenic agars when processing yeastpositive blood cultures and perform antifungal susceptibility testing on all NAC species, especially in centers that still favor fluconazole as the first-line therapy for candidemia.

\section{ACKNOWLEDGMENT}

We would like to thank the Dean of the Faculty of Medicine, Universiti Kebangsaan Malaysia, for her motivation and permission to publish this article.

\section{AUTHORS' CONTRIBUTIONS}

Study conception and design: Chuan Hun Ding. Collection and data analysis: Zaili Zaki. Drafting of article and revising it critically for important intellectual content: Chuan Hun Ding and Zaili Zaki. Final approval of the version to be submitted: Chuan Hun Ding and Zaili Zaki. 


\section{CONFLICTS OF INTEREST}

The authors declare that they have no conflicts of interest.

\section{REFERENCES}

1. Delaloye J, Calandra T. Invasive candidiasis as a cause of sepsis in the critically ill patient. Virulence 2014;5:161-9.

2. Wisplinghoff $H$, Seifert H, Wenzel RP, Edmond MB. Inflammatory response and clinical course of adult patients with nosocomial bloodstream infections caused by Candida spp. Clin Microbiol Infect 2006;12:170-7.

3. Kaur R, Dhakad MS, Goyal R, Kumar R. Emergence of non-albicans Candida species and antifungal resistance in intensive care unit patients. Asian Pac J Trop Biomed 2016;6:455-60.

4. Krcmery V, Barnes AJ. Non-albicans Candida spp. Causing fungaemia: Pathogenicity and antifungal resistance. J Hosp Infect 2002;50:243-60.

5. Kothavade RJ, Kura MM, Valand AG, Panthaki MH. Candida tropicalis: Its prevalence, pathogenicity and increasing resistance to fluconazole. J Med Microbiol 2010;59:873-80.

6. Zhao Y, Chan JF, Tsang CC, Wang H, Guo D, Pan Y, et al. Clinical characteristics, laboratory identification, and in vitro antifungal susceptibility of Yarrowia (Candida) lipolytica isolates causing fungemia: A multicenter, prospective surveillance study. J Clin Microbiol 2015;53:3639-45.

7. Ding $\mathrm{CH}$, Wahab AA, Muttaqillah NA, Tzar MN. Prevalence of albicans and non-albicans candiduria in a Malaysian medical centre. J Pak Med Assoc 2014;64:1375-9.

8. Jensen J, Muñoz P, Guinea J, Rodríguez-Créixems M, Peláez T, Bouza E, et al. Mixed fungemia: Incidence, risk factors, and mortality in a general hospital. Clin Infect Dis 2007;44:e109-14.

9. Al-Rawahi GN, Roscoe DL. Ten-year review of candidemia in a canadian tertiary care centre: Predominance of non-albicans Candida species. Can J Infect Dis Med Microbiol 2013;24:e65-8.

10. Pulimood S, Ganesan L, Alangaden G, Chandrasekar P. Polymicrobial candidemia. Diagn Microbiol Infect Dis 2002;44:353-7.

11. Pappas PG, Kauffman CA, Andes DR, Clancy CJ, Marr KA, OstroskyZeichner L, et al. Clinical practice guideline for the management of candidiasis: 2016 update by the Infectious Diseases Society of America. Clin Infect Dis 2016;62:e1-50.

12. Dharajiya D, Jasani H, Khatrani T, Kapuria M, Pachchigar K, Patel P. Evaluation of antibacterial and antifungal activity of fenugreek (Trigonella foenum-graecum) extracts. Int J Pharm Pharm Sci 2016;8:212-7.

13. Pandey S. Antibacterial and antifungal activities of Ocimum gratissimum L. Int J Pharm Pharm Sci 2017;9:26-31. 